\title{
Measurement ofTLR-induced macrophage spreading by automated image analysis: differential role of Myd88 and MAPK in early and late responses
}

\author{
Jens Wenzel ${ }^{1}$, Christian Held ${ }^{2}$, Ralf Palmisano ${ }^{3}$, Stefan Teufel ${ }^{4}$, Jean-Pierre David ${ }^{4}$, Thomas Wittenberg ${ }^{2}$ and \\ Roland Lang ${ }^{1}$ * \\ 1 Immunology and Hygiene, Institute of Clinical Microbiology, University Hospital Erlangen, Friedrich-Alexander Universität Erlangen-Nürnberg, Erlangen, Germany \\ 2 Department of Image Processing and Biomedical Engineering, Fraunhofer Institute for Integrated Circuits IIS, Erlangen, Germany \\ ${ }^{3}$ Department of Biology, Friedrich-Alexander Universität Erlangen-Nürnberg, Erlangen, Germany \\ ${ }^{4}$ Medical Clinic 3, University Hospital Erlangen, Friedrich-Alexander Universität Erlangen-Nürnberg, Erlangen, Germany
}

Edited by:

Kumar Selvarajoo, Keio University, Japan

Reviewed by:

Sangdun Choi, Ajou University, South Korea

Konrad Alexander Bode, University of Heidelberg, Germany

\section{*Correspondence:}

Roland Lang, Immunology and

Hygiene, Institute of Clinical

Microbiology, University Hospital

Erlangen,

Friedrich-Alexander-Universität

Erlangen-Nürnberg, Wasserturmstr.

3-5, 91054 Erlangen, Germany.

e-mail: roland.lang@uk-erlangen.de
Sensing of infectious danger by toll-like receptors (TLRs) on macrophages causes not only a reprogramming of the transcriptome but also changes in the cytoskeleton important for cell spreading and motility. Since manual determination of cell contact areas from fluorescence micrographs is very time-consuming and prone to bias, we have developed and tested algorithms for automated measurement of macrophage spreading. The two-step method combines identification of cells by nuclear staining with DAPI and cell surface staining of the integrin CD11b. Automated image analysis correlated very well with manual annotation in resting macrophages and early after stimulation, whereas at later time points the automated cell segmentation algorithm and manual annotation showed slightly larger variation. The method was applied to investigate the impact of genetic or pharmacological inhibition of known TLR signaling components. Deficiency in the adapter protein Myd88 strongly reduced spreading activity at the late time points, but had no impact early after LPS-stimulation. A similar effect was observed upon pharmacological inhibition of MEK1, the kinase activating the mitogen-activated protein kinases (MAPK) ERK1/2, indicating that ERK1/2 mediates Myd88-dependent macrophages spreading. In contrast, macrophages lacking the MAPK p38 were impaired in the initial spreading response but responded normally $8-24 \mathrm{~h}$ after stimulation. The dichotomy of $\mathrm{p} 38$ and ERK1/2 MAPK effects on early and late macrophage spreading raises the question which of the respective substrate proteins mediate(s) cytoskeletal remodeling and spreading. The automated measurement of cell spreading described here increases the objectivity and greatly reduces the time required for such investigations and is therefore expected to facilitate larger throughput analysis of macrophage spreading, e.g., in siRNA knockdown screens.

Keywords: macrophage, spreading, TLR, image analysis

\section{INTRODUCTION}

Macrophages reside in all tissues and play an important role in tissue remodeling and homeostasis by phagocytosis and digestion of dead cells and cellular debris. Their second function as sentinels for infectious danger is embodied by the expression of pattern recognition receptors for pathogen-associated molecular patterns. The best characterized group of PRR is the toll-like receptor (TLR) family, which includes TLR9 as the receptor for CpG-rich bacterial DNA and TLR4, which together with MD2 forms the receptor for the lipopolysaccharide from the cell wall of Gram-negative bacteria (Kawai and Akira, 2010). Triggering of TLR family members by microbial ligands, e.g., during phagocytosis of bacteria, induces a rapid and massive transcriptional response engendering the inflammatory response to infection. A bottleneck in the signal transduction of TLR is the adapter protein Myd88 that binds to the intracellular TIR domain of most TLR and recruits further adapters (e.g., TRAF6) and kinases (e.g.,
IRAK1; IRAK4; Kawai et al., 1999; Kawai and Akira, 2010). The major signaling modules activated by TLR are the IKK complex leading to NFKB translocation to the nucleus, and the cascade of mitogen-activated protein kinases (MAPK). MAPK family members expressed in macrophages are ERK1/2, JNK1/2, and p38. These MAPK control the transcriptional response to TLR ligation through phosphorylation-mediated activation of transcription factors (e.g., AP-1, CREB, and many others; Lang et al., 2006); in addition, the plethora of MAPK substrate proteins are involved in diverse cellular processes including cell motility, adhesion, and phagocytosis (Schmidt et al., 2001; Blander and Medzhitov, 2004; West et al., 2004; Kang et al., 2008).

In a recent global and quantitative analysis of TLR4-driven phosphorylation events in primary macrophages, we identified more than 1800 phosphoproteins containing nearly 7000 phosphorylation sites. LPS-stimulation caused reproducible changes in the phosphorylation of around a quarter of all sites both early 
$\left(15^{\prime}\right)$ and late $(4 \mathrm{~h})$ after stimulation (Weintz et al., 2010). Bioinformatic analysis of the regulated phosphoproteome showed an enrichment of known TLR signaling pathway molecules, but also revealed that genes annotated in Gene Ontology as cytoskeletaland actin-binding proteins were enriched and thus are a hotspot of TLR-induced phosphorylation. The set of cytoskeleton-associated proteins showing phosphorylation after TLR4 triggering includes (among others) adapters like Arp3, Paxillin and Vasp, kinases like Ptk2, and motor proteins like Myole, Myolf, and Myo9b. Some of these proteins possess well characterized functions in phagocytosis, cell spreading and motility in macrophages, or other cell types (Schaller, 2001; Sechi and Wehland, 2004; Kim et al., 2006; Hanley et al., 2010). Others have not yet been associated functionally with cytoskeleton-based macrophage responses.

In order to better understand the control of TLR-driven changes in phagocytosis and macrophage spreading by signaling molecules and components of the cytoskeleton, quantitative readout systems are needed. Ideally, such assay systems should have the potential for high-throughput analysis, for example to test the effects of siRNA knockdown of a larger group of candidate genes. The spreading response of cells to stimulation increases the contact area with the cell culture support material, an effect that can be easily visualized in fluorescence microscopy. Quantitation of spreading responses from many cells can be done manually, e.g., using ImageJ software (Girish and Vijayalakshmi, 2004; Collins, 2007). However, this approach has the drawback of being very labor-intensive and time-consuming, hence excluding the analysis of multiple samples in a reasonable amount of time. In addition, the manual delineation of the cell borders introduces potential bias. In contrast, automatic image analysis has the potential of circumventing these drawbacks and is therefore highly desirable. Available freeware software packages like the so-called CellProfiler (Carpenter et al., 2006) are able to perform an automated image analysis. The software can be adapted to the image content by adjusting the minimum and maximum cell diameter and by choosing a threshold selection method. Furthermore various pre-processing methods exist that can improve image quality. However, the requirement to adjust all these parameters until satisfying results are obtained makes the use very time-consuming for researchers not trained in image processing. Thus, we have developed algorithms to segment nuclei and to determine cell size and contact areas (Held et al., submitted; Held et al., 2011). Here, these methods were applied and optimized to investigate the macrophage spreading in response to TLR4 stimulation and its control by the Myd88 adapter protein and the MAPK family members ERK1/2 and $\mathrm{p} 38$.

\section{MATERIALS AND METHODS \\ MICE}

Colonies of C57BL/6, Myd88-/- (Kawai et al., 1999), and p38flox/flox; Mx-Cre (Engel et al., 2005; Bohm et al., 2009) mice were maintained at the Franz Penzoldt Center of the Medical Faculty at the University Erlangen-Nuremberg. p38flox/flox; MxCre mice were injected three times i.p. at week 5 with poly I:C (13 mg/kg body weight) for depletion of p38a in hematopoietic cells as described (Bohm et al., 2009).

\section{REAGENTS}

LPS (Escherichia coli 0111:B4) and Cytochalasin D were purchased from Sigma-Aldrich. MEK1 inhibitor was received from Selleck Chemicals. Antibodies to phosphorylated (p)-Erk1/2, p38, p-p38, pMAPKAPK2 (p-MK2) were obtained from Cell Signaling Technology and to Grb2 from BD Biosciences.

\section{MACROPHAGE DIFFERENTIATION}

Mouse bone marrow was flushed out of the prepared femurs and tibiae with sterile ice-cold PBS. After $5 \mathrm{~min}$ of erythrocyte lysis at room temperature with $\mathrm{NH}_{4} \mathrm{CL}[0.15 \mathrm{M}]$ cells were washed in complete Dulbecco's modified Eagle's medium (cDMEM; $0.05 \mathrm{mM} \beta$-mercapthoethanol, $1 \%$ Pen/Strep; $10 \%$ FCS). After overnight depletion of adherent cells non-adherent cells were further incubated in cDMEM containing 10\% L-cell conditioned medium (LCCM) as a source of M-CSF for 6 days on $10 \mathrm{~cm}$ bacteriological plates at $37^{\circ} \mathrm{C}, 5 \% \mathrm{CO}_{2}$. After 3 days in between $5 \mathrm{ml}$ of cDMEM $+10 \%$ LCCM was added to the cell culture.

\section{STIMULATION OF MACROPHAGES}

Differentiated macrophages were washed with $10 \mathrm{ml}$ sterile PBS, incubated with accutase, collected and washed twice with CDMEM. Macrophages were seeded at a density of $5 \times 10^{4}$ and $2.5 \times 10^{4}$ cells/well on eight-well Permanox chamber slides (Nalge Nunc International). After an overnight cultivation at $37^{\circ} \mathrm{C}$ cells were stimulated with $100 \mathrm{ng} / \mathrm{ml}$ LPS for different time points and finally fixed for $20 \mathrm{~min}$ with $2 \%$ PFA. Supernatants of stimulated cells were collected for examination of cytokine secretion by ELISA.

\section{WESTERN BLOT}

To determine the efficiency of MEK1 inhibitor and p38 deletion, parallel cultures with $1 \times 10^{6}$ cells/well were cultured in $1 \mathrm{ml}$ cDMEM in 12-well plates. Cell lysates were prepared at the indicated time points after LPS-stimulation. $25 \mu \mathrm{l}$ of lysate were loaded on $10 \%$ PAA gel containing SDS. After blotting membrane was blocked in TBS buffer containing 3\% BSA and $0.01 \%$ Tween 20 and proteins were detected with the respective antibodies.

\section{ELISA}

Supernatants from the spreading experiments were collected from the respective conditions and cytokine concentration was measured by ELISA kits from R\&D. Samples were treated as described in the manufacturer's instructions.

\section{STAINING PROCEDURE}

For visualization of the cell surface by fluorescence microscopy, macrophages were incubated for 30-45 $\mathrm{min}$ at room temperature with $1 \mu \mathrm{g} / \mathrm{ml}$ Allophycocyanin (APC)-labeled anti-mouse CD11b antibody (BioLegends) in PBS containing 2\% FCS. Cells were washed twice with PBS and nuclei were stained by addition of $1 \mu \mathrm{g} / \mathrm{ml}$ DAPI (Sigma D8417) in PBS and incubation of $10 \mathrm{~min}$ at room temperature. For further sample preparation cells were washed again with PBS, mounted with 70\% glycerol on specimen and covered. 


\section{IMAGE ACQUISITION AND PROCESSING}

Images were acquired using a Zeiss AxioVert 200M (Germany) widefield microscope connected to an AxioCam MRm camera. Images were taken using a $20 \times$ objective and post-processed by contrast and brightness enhancement within the AxioVison 4.8.2 software (Carl Zeiss MicroImaging). CD11b-APC stained macrophages were imaged using a CY5 filter set from AHF (Germany).

\section{ALGORITHM DESCRIPTION}

Staining of the cell nuclei facilitates the unequivocal definition of individual cells in macrophage cultures containing cells in contact with each other. This information about the nuclei was also included for automatic segmentation, i.e., the definition of macrophage cell bodies against the background and from other cells. The resulting two-step segmentation method consists of the segmentation of all cell nuclei based on the DAPI stain, followed by the segmentation of the macrophages according to the CD11b cell surface signal.

\section{SEGMENTATION AND SEPARATION OF NUCLEI}

For the automatic segmentation of the nuclei, a watershed transform-based segmentation routine (Roerdink and Meijster, 2000) was applied. For pre-processing, Gaussian smoothing was applied to reduce the noise level in the image. After shading correction, an additional pre-processing filter is applied which facilitates the splitting of touching cell nuclei. This algorithm will be described in detail elsewhere (Held et al., submitted). In brief, this filter uses adaptive weighting of the local principal curvature $C_{\mathrm{P}}$. As boundaries between touching cells usually hold $C_{\mathrm{P}}>0$, intensities of these pixels are reduced while other pixels are preserved. The result of this operation is denoted as modified curvature $C_{\mathrm{P}}^{-}$:

$C_{\mathrm{p}}^{-}= \begin{cases}C_{\mathrm{p}}, & \text { if } C_{\mathrm{p}}>0 \\ 0, & \text { else }\end{cases}$

After normalization to the range of $[0,1]$ the modified curvature $C_{\mathrm{P}}^{-}$can be used as a weight map for the input image $I$, yielding a filtered image $I_{\mathrm{F}}$ :

$I_{\mathrm{F}}=\frac{\alpha+\left(1-C_{\mathrm{P}}^{-}\right)^{\mathrm{p}}}{1+\alpha}$

where the parameters $\alpha$ and $p$ determine the strength of the applied filter. After filtering, the nuclei are separated from the image background by a k-means clustering-based threshold selection method (Hartigan and Wong, 1979). Holes in the resulting binary image were filled and a distance transform (Saito and Toriwaki, 1994) was applied to incorporate prior knowledge on the morphology of the nuclei. For separation of nuclei touching each other a watershed transform was applied to the distance image.

\section{SEGMENTATION OF CELLS}

After segmentation of the cell nuclei, the contact area of the macrophage with the slide was segmented. Details on this algorithm have been described elsewhere (Held et al., 2011). In brief, analog to the nuclei image, the macrophage image was smoothed with a Gaussian filter and a shading correction was performed. Afterward, the cells were separated from the image background by application of the k-means clustering algorithm. Note that a different number of clusters was used for the separation of "background and cells" and "background and nuclei." For separation of the cells a gradient magnitude based fast marching level set method (Sethian, 1999) was performed, using the segmented cell nuclei as initialization.

\section{STATISTICAL ANALYSIS}

If not described otherwise in the figure legend, results were expressed as means \pm SEM of at least 300 cells per condition. Graphs were generated with GraphPad Prism and statistical significance was determined with Student $t$ test for unpaired conditions (ns = not significant; ${ }^{*} p<0.05 ;{ }^{* *} p<0.0001$ ).

\section{RESULTS}

To quantitatively measure spreading responses of macrophages to TLR stimulation, the contact area of the cell to the support material needs to be determined. In order to define the cell borders against the background of the slide, we tested different staining approaches for the best discrimination. In our hands, using bone marrow derived mouse macrophages cultured on Permanox chamber slides, the staining of the integrin CD11b with an APC-labeled antibody resulted in more even staining than the lipid-staining molecule $\mathrm{PKH}$ and in a better signal-to-noise ratio than the cytosolic dye CFSE (data not shown). Therefore, CD11b staining of the macrophage cell surface was used for definition of the contact area to the slide and combined with a DAPI staining of the cell nuclei to clearly identify individual cells on fluorescent microscopy images (Figure 1A,B). The spreading response of macrophages to stimulation with the TLR4 agonist LPS was examined in a kinetic analysis using time points between $1 \mathrm{~h}$ and $24 \mathrm{~h}$ (Figure 1C). A small but significant increase in the contact area of $10-15 \%$ was observed already $1 \mathrm{~h}$ after addition of $100 \mathrm{ng} / \mathrm{ml}$ LPS. Over time, macrophages continued to spread on the Permanox surface and extended the contact area to approximately twice the initial size after 8-24 h. In most experiments, the maximum effect was observed at $24 \mathrm{~h}$; in some experiments, a peak was reached already $8 \mathrm{~h}$ after LPS-stimulation (see below). The increase in the macrophage contact area with the Permanox surface was completely prevented when the inhibitor of actin polymerization Cytochalasin D was added to the cultures before addition of LPS (Figure 1C).

The manual annotation of contact areas from the fluorescence microscopy images is very time-consuming, prohibiting the performance of experiments with multiple conditions. Therefore, we applied the automated two-step segmentation algorithm described in the Section "Materials and Methods" to the raw image data from resting and LPS-stimulated macrophages (Figure 2). The results of the automated segmentation are displayed by the software and the annotation of the cell borders is highlighted (Figure 2B), allowing quality control and manual editing by the user (Figure 2C). While for most cells, the segmentation obtained by the software was found to be correct upon inspection, in the case of overlapping cells some manual editing of the contact area annotation was required (arrows in Figure 2C). 


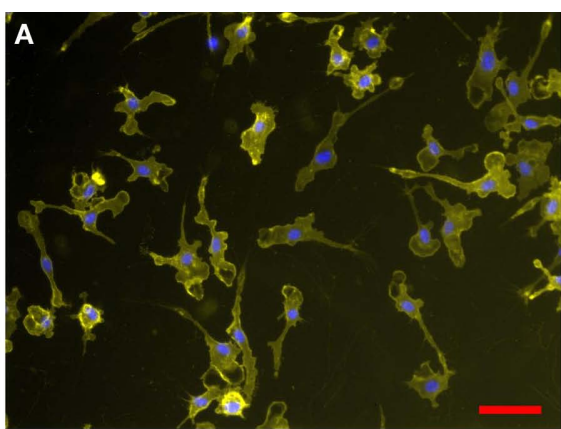

control

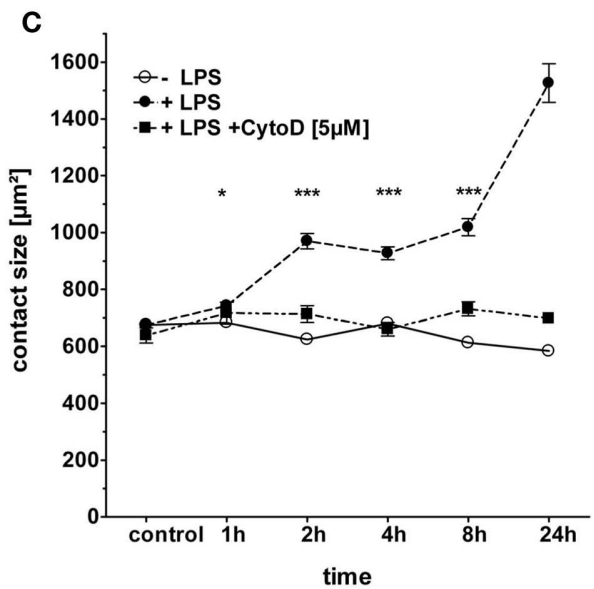

FIGURE 1 | LPS-induced spreading of macrophages. BMM were plated in eight-well chamber slides and rested overnight before addition of LPS

$(100 \mathrm{ng} / \mathrm{ml})$. The inhibitor of actin polymerization Cytochalasin D $(5 \mu \mathrm{g} / \mathrm{ml})$ was added $1 \mathrm{~h}$ prior to LPS-stimulation. At the indicated time points after addition of LPS, slides were processed for staining of CD11b and nuclei, followed by fluorescence microscopy. (A,B) Representative images from control and $24 \mathrm{~h}$

To test the accuracy and reliability of the automatic segmentation algorithm, we directly compared the distribution of contact areas on a large number of macrophages under resting and LPS-activated conditions using automated segmentation by the software tool versus manual annotation as the gold standard (Figure 3A). In this comparison based on the image data from a single experiment, both methods yielded very similar distribution of contact areas and median values in resting cells and early after LPS-stimulation. At the later timepoints, the automatic segmentation algorithm produced a "shrinkage effect" with a smaller median value compared to the manual annotation. To determine whether this "shrinkage effect" is generic at later timepoints, we extended this comparison analysis to a series of six independent experiments (Figure 3B). Comparison of the median contact area values shows a consistent high agreement between the automated method and the manual annotation in resting macrophages and early $(2 \mathrm{~h})$ after stimulation. At the later timepoints, the differences obtained by both methods tended to become larger; however, in addition to "shrinkage effects" there were also "blow-up effects," i.e., examples where automatic annotation gives larger values compared to the manual annotation method. Overall, we observed a

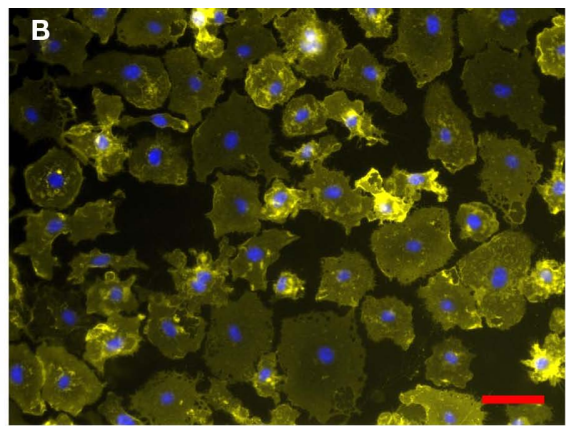

24h LPS time points. Scale bar $=50 \mu \mathrm{m}$. (C) Quantitation of cell spreading by manual annotation of CD11b staining. Shown are mean and SEM of at least 300 cells per condition from one representative experiment. Media control (open circles), LPS (closed circles), and LPS in the presence of Cytochalasin D (closed squares). Statistical significance refers to LPS-treated compared to untreated condition. ns $=$ not significant; ${ }^{*} p<0.05 ;{ }^{* *} p<0.0001$. good agreement between the results obtained by automated segmentation and manual annotation even at the later timepoints. Therefore, we employed the automated method to investigate TLRtriggered macrophage spreading and its control by canonical TLR pathway molecules; for comparison and validation of the method, the time-consuming manual editing of the automatic annotated data was included in each experiment.

Most TLR employ the adapter protein Myd88 for activation of the major signaling pathways leading to gene expression and cytokine secretion. The MAPK p38 and ERK1/2 are activated by TLR stimuli and contribute to cellular responses through phosphorylation of transcription factors and other substrate proteins. We employed macrophages with genetic deletion of Myd88 and p38, and pharmacological inhibition of the ERK1/2 kinase MEK1, to determine the control of LPS-induced macrophage spreading by these molecules. The effect of genetic and pharmacologic deletion on the cytokine response to LPS was examined using supernatants from the cultures used for analysis of cell spreading (Figure 4). In the absence of Myd88, secretion of the pro-inflammatory TNF, IL-6, and IL-12p40, as well as of anti-inflammatory IL-10 was almost completely absent. In contrast, the inhibition or genetic 

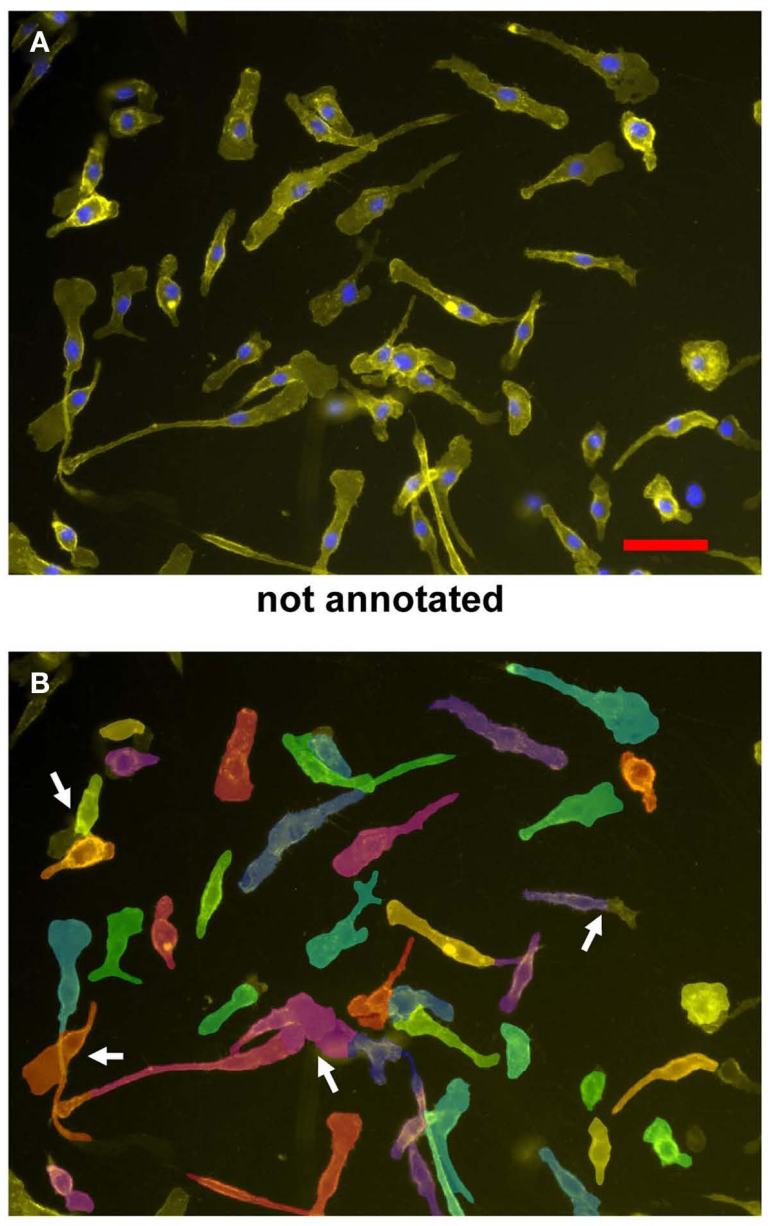

tool annotated

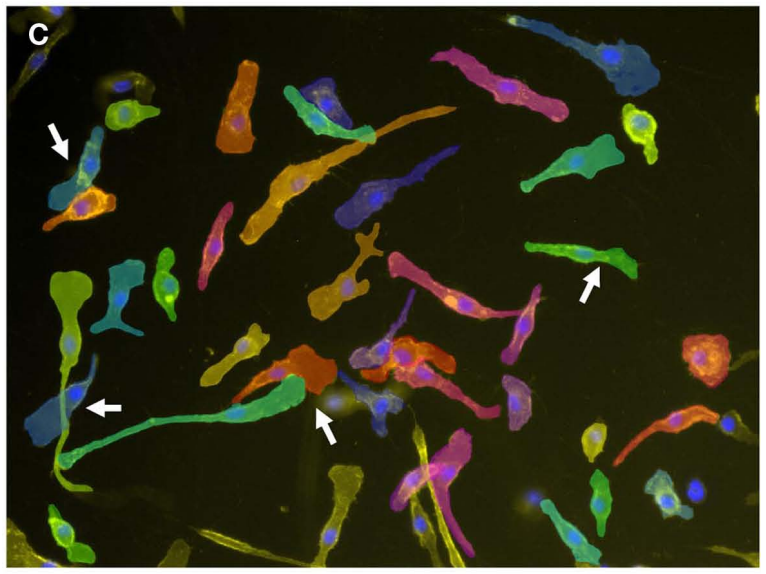

user corrected

FIGURE 2 | Segmentation and annotation of macrophages with two-step segmentation software. Fluorescence microscopy images of CD11b-APC- and DAPI-stained macrophages were uploaded into the software (A), nuclear segmentation and contact area annotation was performed by the software tool (B), and finally checked and corrected by the user (C). Arrowheads indicate shrinkage effects; arrows point to overlapping cells necessitating manual editing of the annotation. Cells were imaged by a $20 \times$ objective. Scale bar represents $50 \mu \mathrm{m}$. ablation of the MAPK family members had more subtle effects. TNF is secreted rapidly after LPS-stimulation; its levels were moderately (40-60\%) decreased by the MEK1 inhibitor PD184352 or in p38-deficient macrophages throughout the time course (Figure 4A). In contrast, IL-6 is secreted later and was less affected by interference with MAPK signals (Figure 4B). Of interest, IL12 p40 was increased upon inhibition of MEK1 or in p38-deficient macrophages (Figure 4C). IL-10 production was severely impaired in the absence of $\mathrm{p} 38$, but also blocked considerably by MEK1 inhibition (Figure 4D).

We next determined the spreading response of macrophages after LPS-stimulation in the absence of Myd88 (Figure 5). In a comparison of Myd88 heterozygous and knockout macrophages, we observed that the strong induction of macrophage spreading at the late 8 and $24 \mathrm{~h}$ timepoints was indeed severely impaired in the absence of Myd88. However, Myd88-/- macrophages did respond nearly as well to LPS at the early timepoint of $2 \mathrm{~h}$ as the heterozygous control cells. Although there were slight differences in the absolute values, this pattern of responsiveness was robustly identified by both segmentation methods (left and right panel in Figure 5). Myd88-/- macrophages showed a complete lack of cytokine secretion when supernatants from the chamber slide cultures were examined by ELISA (Figure 4). The conserved early spreading response in the absence of Myd88 is therefore likely independent of cytokine secretion. Furthermore, it indicates that other signaling molecules (e.g., the adapter TRIF) may play an important role in cytoskeletal rearrangement after LPS.

The second generation MEK1 inhibitor PD184352 has been reported to have a higher specificity than the older reagents U0126 and PD98059 for MEK1 over MEK5 (Grill et al., 2004), and was therefore used here. Pretreatment of macrophages with PD184352 strongly reduced the levels of basal and LPS-induced phosphorylation of ERK1/2 without obvious impact on the phosphorylation of p38 MAPK (Figure 6A). MEK1 inhibition had no effect on basal macrophage spreading, and did not change the significant spreading observed at the $2 \mathrm{~h}$ timepoint (and in the single experiment also at the $1 \mathrm{~h}$ and $4 \mathrm{~h}$ timepoints). However, the further increase in spreading at the $8 \mathrm{~h}$ and $24 \mathrm{~h}$ timepoints was prevented; in fact, similar to the effect of Myd88 deficiency, MEK1 inhibition caused a reduction of the macrophage contact area between $8 \mathrm{~h}$ and $24 \mathrm{~h}$ (Figure 6B). Again automatic annotation and manual editing gave basically identical results.

A possible contribution of the p38 MAPK to LPS-induced macrophage spreading was investigated using macrophages derived from the bone marrow of conditional p38flox/flox; MxCre mice treated with poly I:C to induce deletion of $\mathrm{p} 38$. As shown in Figure 7A, deletion of p38 was very efficient in mice expressing Cre; consequently, phospho-p38 was undetectable. In contrast, the early activation of ERK1/2 was unchanged in the absence of p38, whereas at later timepoints an even stronger ERK1/2 activation was apparent. The spreading of macrophages in response to LPS was only moderately affected by the absence of p38 (Figure 7B). At the early $2 \mathrm{~h}$ timepoint, p38-/- macrophages showed a significantly attenuated increase in the contact area. This effect was seen with the automatic annotation method as well as after manual editing of the segmentation results. However, by $8 \mathrm{~h}$ and 24 this difference between p38-/ - and p38+/+ macrophages had nearly vanished 

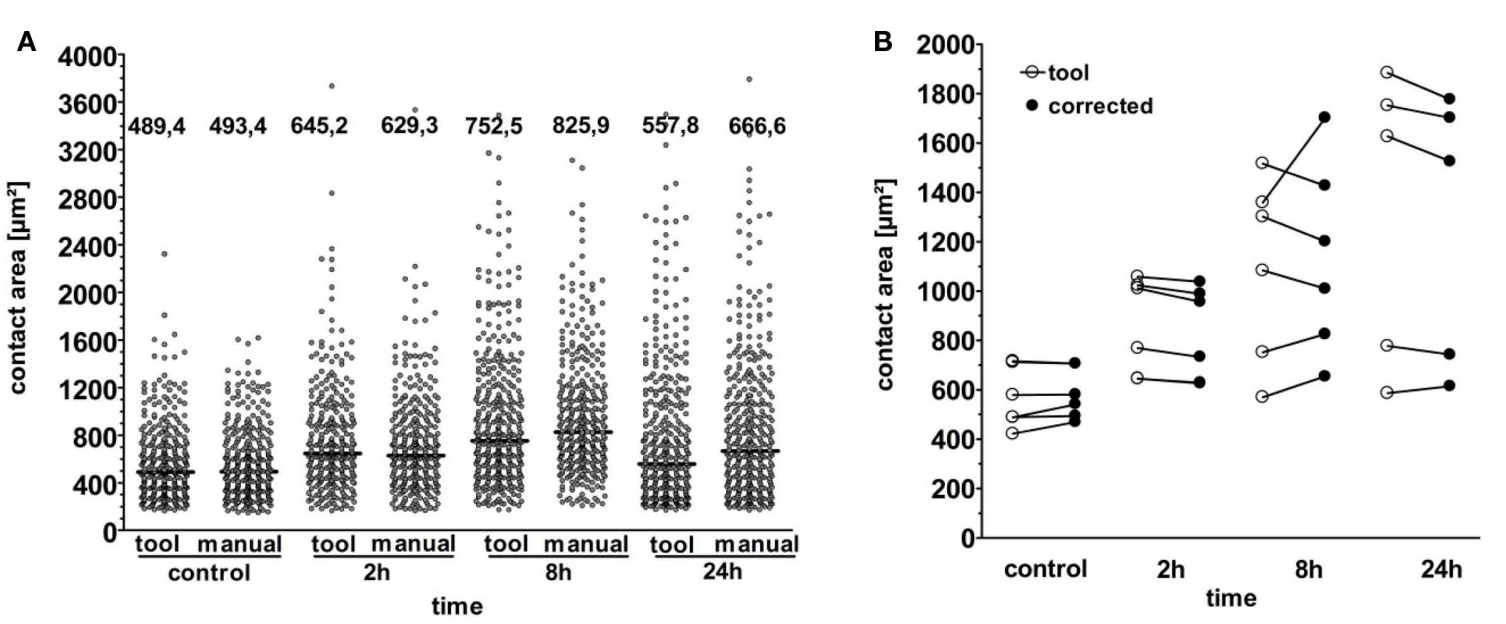

FIGURE 3 | Quantitative comparison of manual versus automatic analysis versus automatic with manual editing. (A) Comparison of cell contact area measured automatically versus manual annotation. Data are from one experiment. Each dot represents one cell. Black bars within the scatter plot show median contact area (numerical value indicated above). (B) Comparison of data obtained by annotation with tool (open circle) versus combination of tool and manual correction (closed circle) from six independent experiments and four experimental conditions. Each dot represents the median of at least 300 annotated cells. and p38-1- macrophages displayed similar spreading behavior as $\mathrm{p} 38+/+$ cells. The $8 \mathrm{~h}$ time point in this experiment is the only instance, where the slight variation between the automated image analysis and the manual editing method led to a different result, in that a significant effect of p38-deficiency was found with the automated annotation but not after manual editing. Together, p38 appears to be required for maximal early spreading, but in marked contrast to the strong effects observed for Myd88 deficiency and inhibition of ERK1/2 activation, p38 is not involved at later timepoints.

\section{DISCUSSION}

In this manuscript, we have described the application of a newly developed algorithm for automated image analysis in the investigation of macrophage spreading in response to TLR stimulation. The rapid acquisition of quantitative and reliable data from microscopy-based assays of changes in cell size and adherence is necessary for the comprehensive investigation of the typically relatively large number of candidate genes identified in systems approaches like transcriptome or proteomic screens. We report here that the algorithm developed, a combination of nuclei separation filter based on a watershed segmentation with subsequent cell segmentation by fast marching level set (Held et al., 2011), performed very well in resting macrophages and early after stimulation; at later timepoints an increase in variation was observed. However, our comparison of the automated algorithm with manually corrected annotations showed that overall very similar results were obtained across several experiments investigating the effects of perturbations in TLR signaling on macrophage spreading. Thus, at least for the relatively strong effects on macrophage spreading observed here, the two-step segmentation method presented and tested here can be used without the need for manual editing of the data, leading to a tremendous reduction in the time and labor required to obtain quantitative data on macrophage spreading.
Hence, using this method medium and high-throughput analysis of macrophage spreading appear feasible. For the investigation of more subtle differences, and for validation of effects found with the automated analysis tool, manual inspection, and editing of the annotation may be required. Of note, even with such a semi-automated method of combining tool-based annotation with manual editing the processing time of the microscopy data is reduced by a factor of two to three compared to manual annotation of cells, thereby enabling the investigation of much larger data sets in a reasonable time frame. Taken together, we are convinced that the method described here represents a considerable technical advance and valuable addition to the toolbox required for quantitative, unbiased, and automatic image analysis of innate immune cells.

To further improve the performance of the automatic segmentation tools several issues should be addressed in future work. First, the quality of the input data, i.e., fluorescence microscopy pictures, in terms of intensity of staining and signal-to-noise ratio of cells versus slide background, appears to be the most critical parameter. We have compared the cytosolic dye CFSE, the membrane lipid stain PKH and APC-labeled anti-CD11b staining (data not shown) and obtained the best results with surface molecule staining by anti-CD11b antibody. The reduced accuracy of the annotation tool at later time points after stimulation may be related to changes in $\mathrm{CD} 11 \mathrm{~b}$ surface expression and/or redistribution of CD11b in different cellular compartments, creating internal maxima that are falsely recognized as cell borders (Figure $\mathbf{2 B}, \mathbf{C}$ ). Thus, staining of other surface markers with strong expression independent of the activation status could lead to enhanced performance of the tool; possible surface markers on macrophages could be MHC-I, CD45, or Fc receptors. In addition, the use of fluorescent dyes yielding stronger signals for labeling of antibodies will be tried to increase signal-to-noise ratios. Another difficulty is the automatic segmentation and annotation of overlapping cells, 


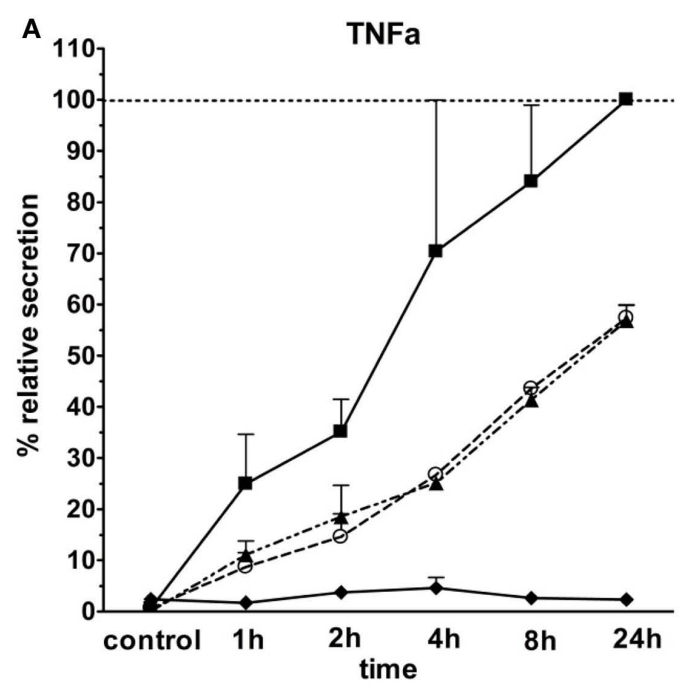

C

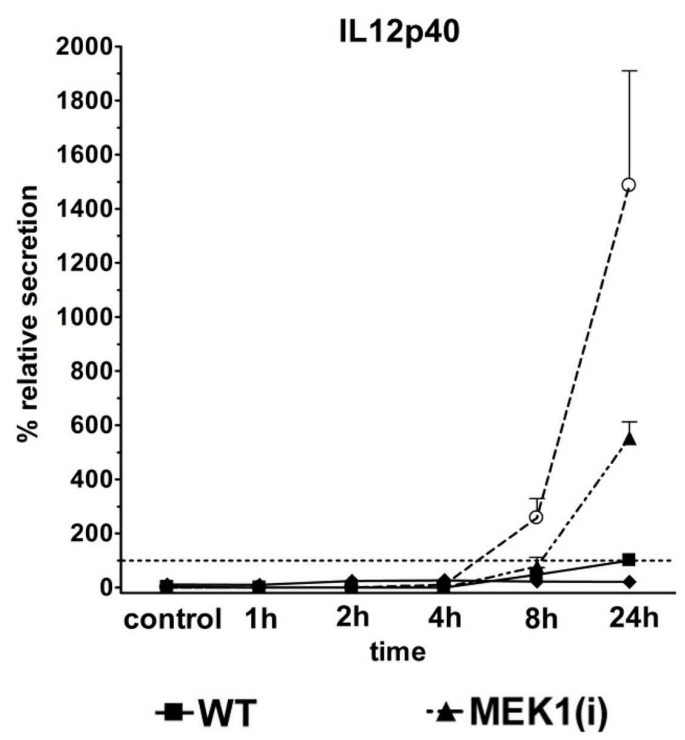

FIGURE 4 | Control of LPS-induced cytokine secretion by Myd88,

ERK1/2, and p38. Supernatants of the cultures used for macrophage spreading analysis were analyzed by ELISA for the presence of the

cytokines TNF (A), IL-6 (B), IL-12p40 (C), and IL-10 (D). To normalize for

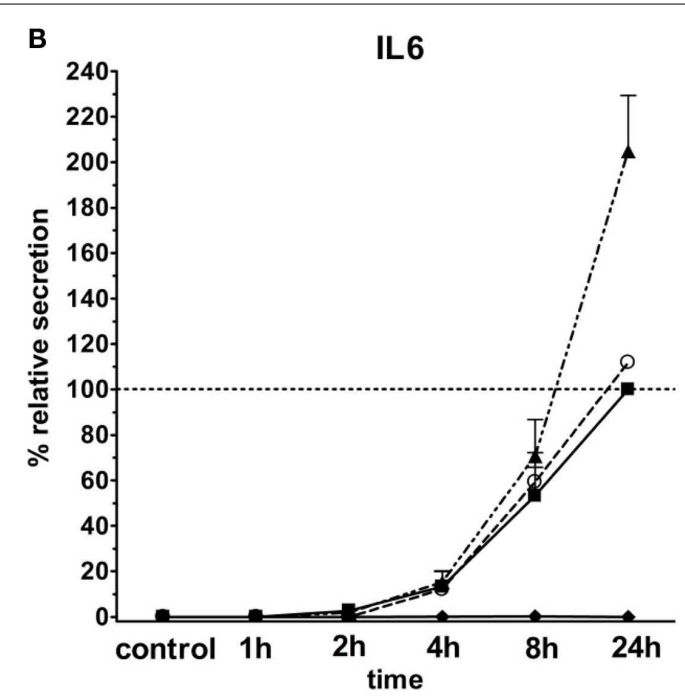

D

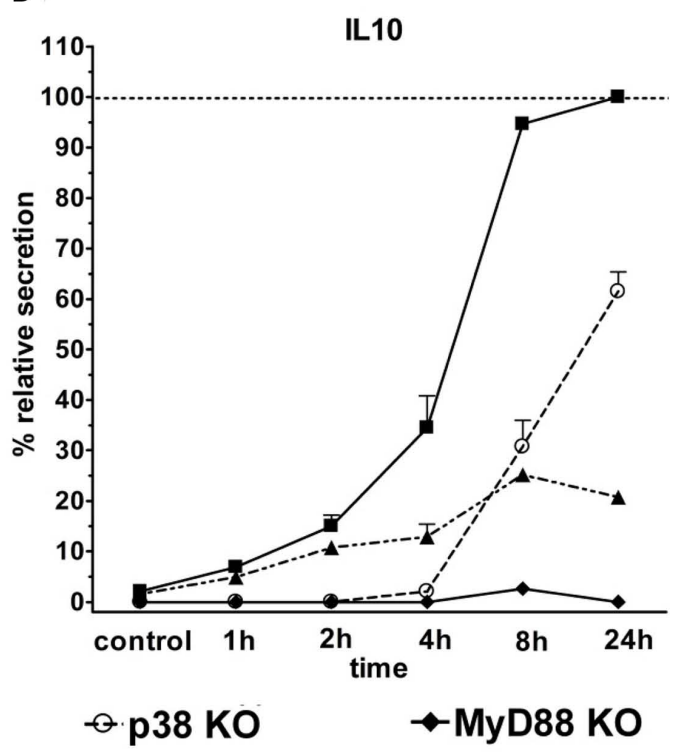

differences between experiments in the absolute amount of cytokines produced, the maximum level of the LPS-treated WT or control macrophage was set to $100 \%$ (shown as -). Shown are mean + SD values from two independent experiments. which may be solved by further development of the segmentation algorithm.

TLR4-triggered macrophage spreading on Permanox slides induced by stimulation with LPS was observed first after $1 \mathrm{~h}$ and increased steadily until $8-24 \mathrm{~h}$. The spreading response could be a consequence of direct TLR-driven signals causing actin polymerization and cytoskeletal rearrangement, or it could depend on indirect effects of TLR-induced secretion of cytokines, e.g., TNF or IL-1. We have not dissected these possibilities here; to do so will require the use of inhibitors of protein synthesis (e.g., Cycloheximide), transport and secretion (e.g., Brefeldin A), or specific interference with certain cytokines by using knockout macrophages. By comparing the kinetics of secretion of the cytokines TNF, IL-6, and
IL-12p40 with the spreading response, we observe that only TNF is produced early enough to have a potential role in the initial spreading response. To assess the role of TNF in macrophage spreading, inhibitors of the metalloprotease Adam17 (also known as TACE) could be used.

The central adapter protein of TLR signaling, Myd88, is required for LPS-induced cytokine production (Figure 4) and for the late and enhanced increase in the contact area; however, the early spreading response at $2 \mathrm{~h}$ was surprisingly normal in Myd88-/- macrophages (Figure 5). To our knowledge, macrophage spreading of Myd88-/- macrophages has not been analyzed before. Since TLR4 utilizes in addition to Myd88 the adapter protein TRIF (Yamamoto et al., 2003; Weighardt et al., 


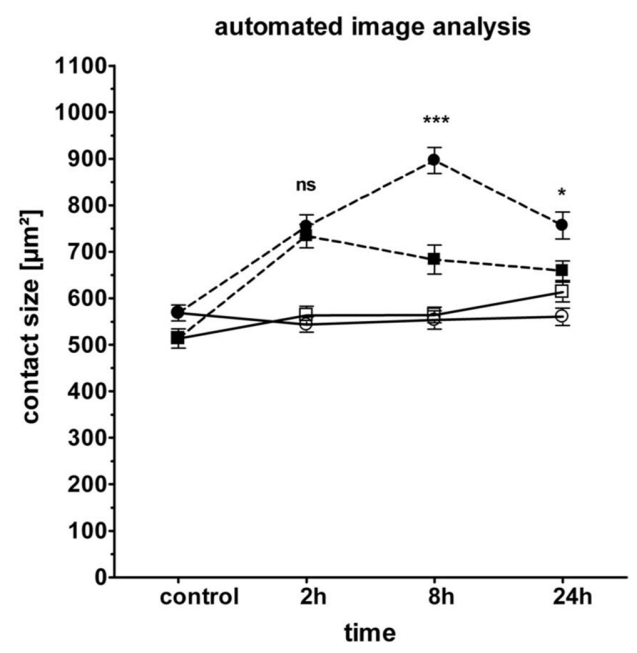

FIGURE 5 | Phenotype of Myd88-/- macrophages in cell spreading. BMM from Myd88 \pm and Myd88-/- mice were plated and stimulated as described in Figure 3. Macrophage spreading was analyzed by automated image analysis (left panel) and manual editing (right panel). Shown are mean

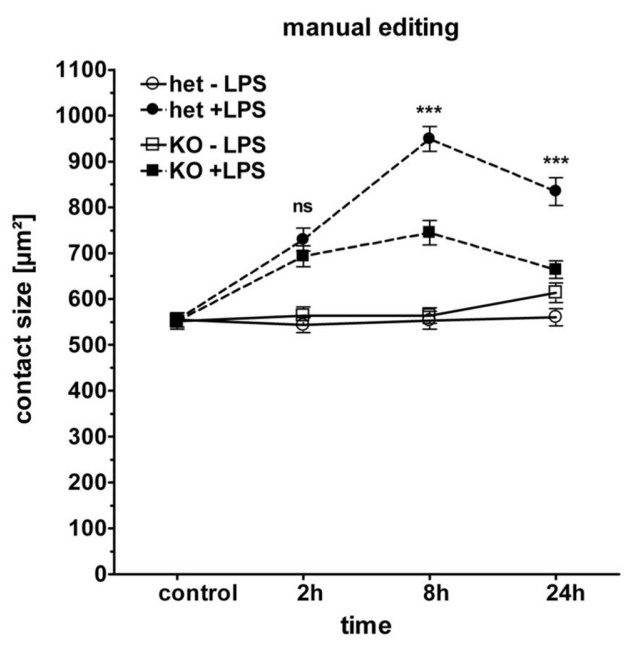

and SEM from one representative experiment of two performed. LPS (closed symbols), media control (open symbol), Myd88 \pm (circles), Myd88-/(squares). Statistical significance refers to Myd88 \pm compared to Myd88-/genotypes. $\mathrm{ns}=$ not significant; ${ }^{* *} p<0.0001$.
A

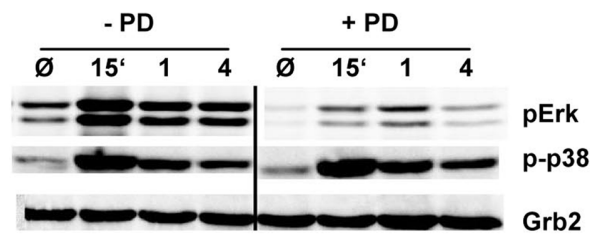

B

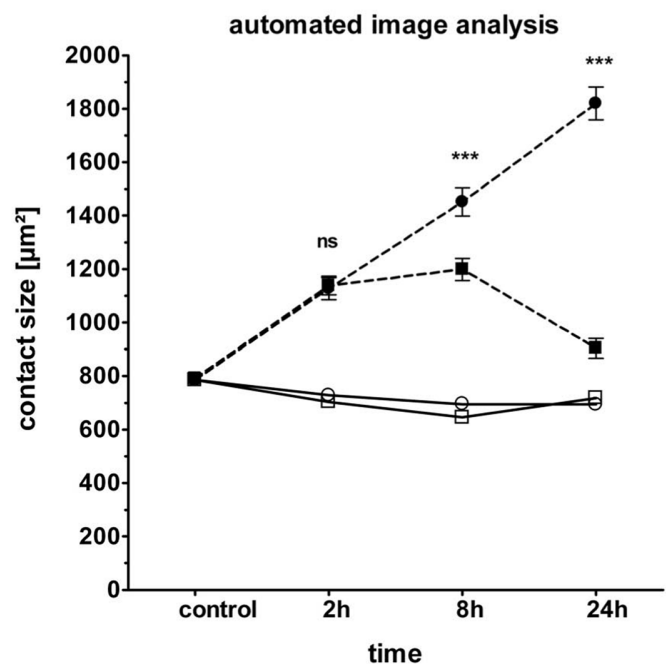

FIGURE 6 | Role of the MAPK ERK in macrophage spreading. BMM from C57BL/6 mice were plated as described in Figure 3. (A) Western blot control for ERK1/2 and p38 phosphorylation. Cell lysates were taken at indicated time points ( $15 \mathrm{~min}$ to $24 \mathrm{~h}$ ) from two individual experiments. Grb2: loading control. (B) Effect of pharmacological blockade of ERK1/2 activation on spreading. Macrophages were pre-incubated with the

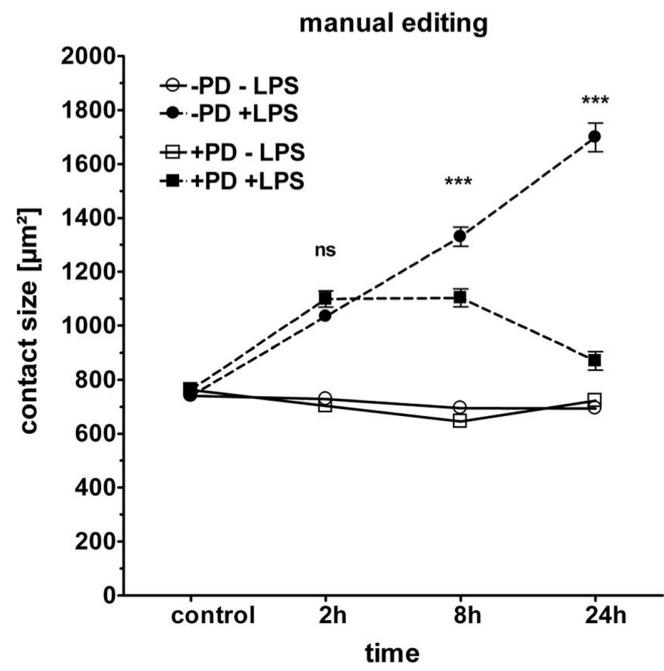

MEK1 inhibitor PD184352 (10 $\mu \mathrm{M}$, squares) or not (circles) for $1 \mathrm{~h}$ prior to addition of LPS (closed symbols) or media control (open symbols). Contact area data obtained by automated image analysis (left panel) and manual editing (right panel). Statistical significance refers to PD184352-treated compared to no inhibitor. ns = not significant; *** $p<0.0001$. 


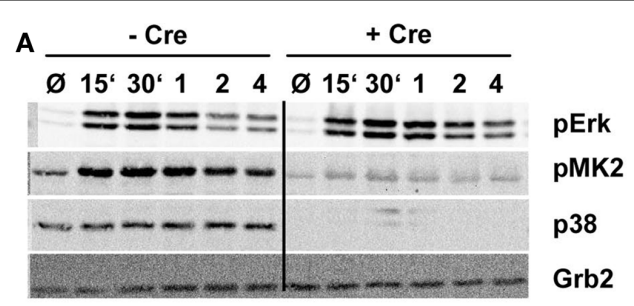

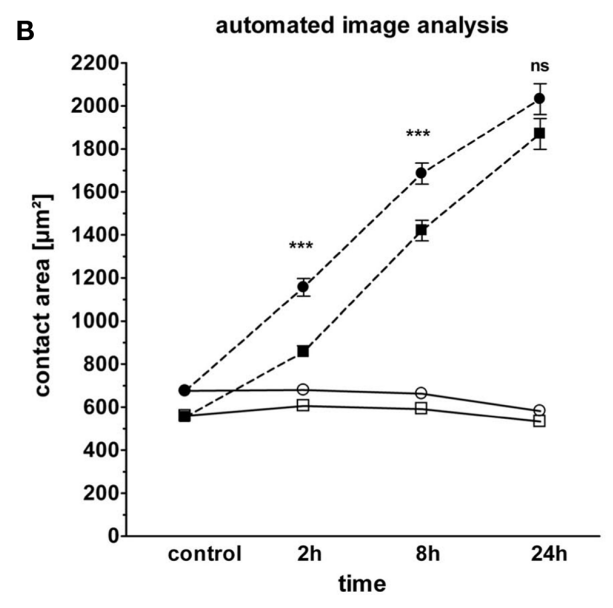

FIGURE 7 | Role of the MAPK p38 in macrophage spreading. Bone marrow from p38flox; $\mathrm{Mx}$-cre; and p38flox mice treated with poly I:C to induce deletion of the p38 gene was used to generate BMM. These cells were plated and stimulated as described in Figure 3. (A) Western blot control for p38 protein levels and phosphorylation of p38 and ERK1/2. Cell lysates were taken at indicated time points ( $15 \mathrm{~min}$ to $4 \mathrm{~h}$ ). Grb2: loading

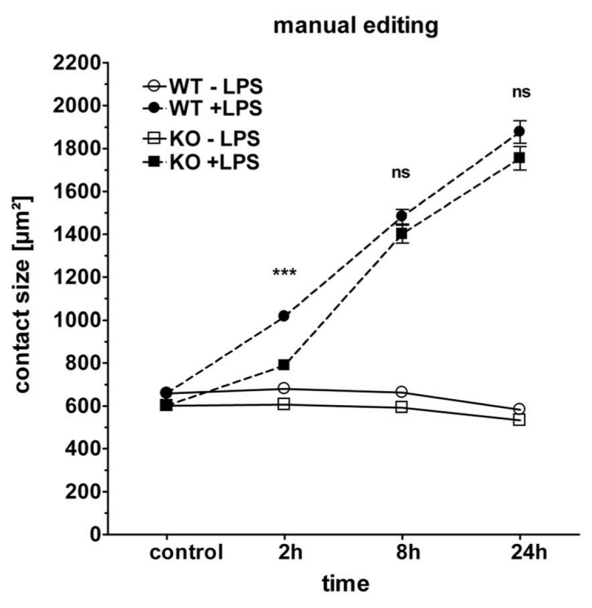

control. (B) Macrophage spreading kinetics of $\mathrm{Cre}+(\mathrm{KO}$, squares) and Cre- (WT, circles) BMM stimulated with LPS (closed symbols) or media control (open symbols). Results of automated image analysis (left panel) and manual editing (right panel). Statistical significance refers to the comparison of p38-deficient versus WT macrophages. ns = not significant; *** $p<0.0001$.

after poly I:C injection. In the absence of p38, a reduced early, but intact late spreading response was found. It is worth mentioning that p38-deficient macrophages, similar to Myd88-/macrophages, had a slightly reduced basal contact area (Figures 5 and 7), which could also indicate differences in macrophage differentiation in the absence of Myd88 and p38. To corroborate the p38-dependence of early LPS-induced spreading, specific pharmacological inhibition of p38 in future experiments will be helpful. Such experiments have already been performed in a macrophage cell line, showing that p38 induces integrin-dependent spreading of J774 macrophages via activation of the Ras-like GTPase Rap1 (Schmidt et al., 2001). As this early defect was not seen in Myd88-deficient macrophages, the logical implication of the data is that $\mathrm{p} 38$ activation should be independent of Myd88. In fact, the LPS-induced phosphorylation of p38 and ERK1/2 MAPK is only somewhat delayed in Myd88-deficient macrophages (Kawai et al., 1999).

Which adapters, GTPases and motor proteins are involved in the LPS-induced spreading response, and how are they controlled by the Myd88- and MAPK-dependent pathways described here? One established pathway from TLR4 to integrin activation is via activation of the focal adhesion kinase-related Pyk2 and the adapter protein paxillin (Williams and Ridley, 2000). Paxillin activation and cell spreading in response to LPS involves ERK1/2dependent phosphorylation of Ser130 which is a prerequisite for 
GSK3-dependent phosphorylation of Ser126 (Cai et al., 2006). All members of the ERK1/2-GSK3-Pyk2-Paxillin module were found strongly phosphorylated after LPS at multiple sites in our published phosphoproteome analysis (Weintz et al., 2010). Of interest, paxillin and Pyk2 activation were only partially reduced in Myd88deficient macrophages (Hazeki et al., 2003), consistent with their potential role in the intact initial spreading in Myd88-deficient macrophages observed here. The observation that $\mathrm{Cdc} 42$ and Rac GTPase activation proceeds independent of Myd88 early after LPS-stimulation (Kong and Ge, 2008) provides another potential mechanism for macrophage spreading. Clearly, while some of the players in macrophage spreading are well established, the regulated phosphorylation of more than 40 proteins with a Gene Ontology annotation of "actin-binding" or "cytoskeleton-binding" (Weintz

\section{REFERENCES}

Blander, J. M., and Medzhitov, R. (2004). Regulation of phagosome maturation by signals from toll-like receptors. Science 304, 1014-1018.

Bohm, C., Hayer, S., Kilian, A., Zaiss, M. M., Finger, S., Hess, A., Engelke, K., Kollias, G., Kronke, G., Zwerina, J., Schett, G., and David, J. P. (2009). The alpha-isoform of p38 MAPK specifically regulates arthritic bone loss. J. Immunol. 183, 5938-5947.

Cai, X., Li, M., Vrana, J., and Schaller, M. D. (2006). Glycogen synthase kinase 3- and extracellular signal-regulated kinase-dependent phosphorylation of paxillin regulates cytoskeletal rearrangement. Mol. Cell Biol. 26, 2857-2868.

Carpenter, A. E., Jones, T. R., Lamprecht, M. R., Clarke, C., Kang, I. H., Friman, O., Guertin, D. A., Chang, J. H., Lindquist, R. A., Moffat, J., Golland, P., and Sabatini, D. M. (2006). CellProfiler: image analysis software for identifying and quantifying cell phenotypes. Genome Biol. 7, R100.

Collins, T. J. (2007). ImageJ for microscopy. BioTechniques 43, 25-30.

Engel, F. B., Schebesta, M., Duong, M. T., Lu, G., Ren, S., Madwed, J. B., Jiang, H., Wang, Y., and Keating, M. T. (2005). p38 MAP kinase inhibition enables proliferation of adult mammalian cardiomyocytes. Genes Dev. 19, 1175-1187.

Girish, V., and Vijayalakshmi, A. (2004). Affordable image analysis using NIH Image/ImageJ. Indian J. Cancer 41, 47.

Grill, C., Gheyas, F., Dayananth, P., Jin, W., Ding, W., Qiu, P., Wang, L., Doll, R. J., and English, J. M. (2004). Analysis of the ERK1,2 transcriptome in mammary epithelial cells. Biochem. J. 381, 635-644.

Hanley, P. J., Xu, Y., Kronlage, M., Grobe, K., Schon, P., Song, J., Sorokin, L., Schwab, A., and Bahler, M. (2010). Motorized RhoGAP myosin IXb
(Myo9b) controls cell shape and motility. Proc. Natl. Acad. Sci. U.S.A. 107, 12145-12150.

Hartigan, J., and Wong, M. A. (1979). A k-means clustering algorithm. J. $R$. Stat. Soc. Ser. A 28, 100-108.

Hazeki, K., Masuda, N., Funami, K., Sukenobu, N., Matsumoto, M., Hazeki, O. (2003). Toll-like receptormediated tyrosine phosphorylation of paxillin via MyD88-dependent and -independent pathways. Eur. J. Immunol. 33, 740-747.

Held, C., Wenzel, J., Webel, R., Marschall, M., Lang, R., Palmisano, R., and Wittenberg, T. (2011). Using multimodal information for the segmentation of fluorescent micrographs with application to virology and microbiology. Conf. Proc. IEEE Eng. Med. Biol. Soc. (in press).

Kang, Y. J., Chen, J., Otsuka, M., Mols, J., Ren, S., Wang, Y., and Han, J. (2008). Macrophage deletion of p38alpha partially impairs lipopolysaccharide-induced cellular activation. J. Immunol. 180, 5075-5082.

Kawai, T., Adachi, O., Ogawa, T., Takeda, K., and Akira, S. (1999). Unresponsiveness of MyD88-deficient mice to endotoxin. Immunity 11, 115-122.

Kawai, T., and Akira, S. (2010). The role of pattern-recognition receptors in innate immunity: update on toll-like receptors. Nat. Immunol. 11, 373-384.

Kim, S. V., Mehal, W. Z., Dong, X., Heinrich, V., Pypaert, M., Mellman, I., Dembo, M., Mooseker, M. S., Wu, D., and Flavell, R. A. (2006). Modulation of cell adhesion and motility in the immune system by Myolf. Science 314, 136-139.

Kong, L., and Ge, B. X. (2008). MyD88-independent activation of a novel actin-Cdc42/Rac pathway is required for toll-like receptorstimulated phagocytosis. Cell Res. 18, 745-755. Akira, S., Takeda, K., Seya, T., and

et al., 2010) indicates that multiple proteins contribute to the changes in macrophage shape, contact area and motility after TLR stimulation. Elucidation of the functional role of these proteins will require siRNA knockdown experiments, macrophages from knockout mice and the use specific pharmacological inhibitors. We believe that the method of semi-automatic measurement of macrophage spreading will greatly facilitate the timely, unbiased and quantitative investigation of these perturbations.

\section{ACKNOWLEDGMENTS}

This project received funding from the Deutsche Forschungsgemeinschaft (SFB 643, TP A10; and SFB 796, B6, A4, and Z). The authors thank Katrin Jozefowski for mouse genotyping, and Matthias Engelbrecht for advice with statistics.

Lang, R., Hammer, M., and Mages, J. (2006). DUSP meet immunology: dual specificity MAPK phosphatases in control of the inflammatory response. J. Immunol. 177, 7497-7504.

Roerdink, J., and Meijster, A. (2000). The watershed transform: definitions, algorithms and parallelization strategies. Math. Morphol. 41, 187-228.

Saito, T., and Toriwaki, J. I. (1994). New algorithms for Euclidean distance transformation of an n-dimensional digitized picture with applications. Pattern Recognit. 27, 1551-1565.

Schaller, M. D. (2001). Paxillin: a focal adhesion-associated adaptor protein. Oncogene 20, 6459-6472.

Schmidt, A., Caron, E., and Hall, A. (2001). Lipopolysaccharideinduced activation of beta2-integrin function in macrophages requires Irak kinase activity, p38 mitogenactivated protein kinase, and the Rap1 GTPase. Mol. Cell Biol. 21, 438-448.

Sechi, A. S., and Wehland, J. (2004). ENA/VASP proteins: multifunctional regulators of actin cytoskeleton dynamics. Front. Biosci. 9, 1294-1310.

Sethian, J. (1999). Level Set Methods and Fast Marching Methods. Cambridge: Cambridge University Press.

Weighardt, H., Jusek, G., Mages, J., Lang, R., Hoebe, K., Beutler, B., and Holzmann, B. (2004). Identification of a TLR4- and TRIF-dependent activation program of dendritic cells. Eur. J. Immunol. 34, 558-564.

Weintz, G., Olsen, J. V., Fruhauf, K., Niedzielska, M., Amit, I., Jantsch, J., Mages, J., Frech, C., Dolken, L., Mann, M., and Lang, R. (2010). The phosphoproteome of toll-like receptor-activated macrophages. Mol. Syst. Biol. 6, 371.

West, M. A., Wallin, R. P., Matthews, S. P., Svensson, H. G., Zaru, R., Ljunggren, H. G., Prescott, A. R., and Watts, C.
(2004). Enhanced dendritic cell antigen capture via toll-like receptorinduced actin remodeling. Science 305, 1153-1157.

Williams, L. M., and Ridley, A. J. (2000). Lipopolysaccharide induces actin reorganization and tyrosine phosphorylation of Pyk2 and paxillin in monocytes and macrophages. J. Immunol. 164, 2028-2036.

Yamamoto, M., Sato, S., Hemmi, H., Hoshino, K., Kaisho, T., Sanjo, H., Takeuchi, O., Sugiyama, M., Okabe, M., Takeda, K., and Akira, S. (2003). Role of adaptor TRIF in the MyD88-independent toll-like receptor signaling pathway. Science 301, 640-643.

Conflict of Interest Statement: The authors declare that the research was conducted in the absence of any commercial or financial relationships that could be construed as a potential conflict of interest.

Received: 08 August 2011; accepted: 21 September 2011; published online: 18 October 2011.

Citation: Wenzel J, Held C, Palmisano $R$, Teufel S, David J-P, Wittenberg T and Lang $R$ (2011) Measurement of TLR-induced macrophage spreading by automated image analysis: differential role of Myd88 and MAPK in early and late responses. Front. Physio. 2:71. doi: 10.3389/fphys.2011.00071

This article was submitted to Frontiers in Systems Physiology, a specialty of Frontiers in Physiology.

Copyright (C) 2011 Wenzel, Held, Palmisano, Teufel, David, Wittenberg and Lang. This is an open-access article subject to a non-exclusive license between the authors and Frontiers Media SA, which permits use, distribution and reproduction in other forums, provided the original authors and source are credited and other Frontiers conditions are complied with. 